\title{
Diagnostic value analysis of combined detection of Trx, CYFRA21-1 and SCCA in lung cancer
}

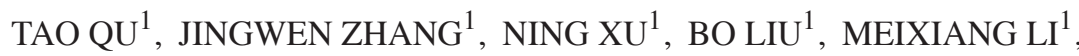 \\ AILING LIU ${ }^{1}$, AIJUN LI ${ }^{1}$ and HUAPING TANG ${ }^{2}$ \\ ${ }^{1}$ Department of Pneumology, Weihai Municipal Hospital, Weihai, Shandong 264200; \\ ${ }^{2}$ Department of Pneumology, Qingdao Municipal Hospital, Qingdao, Shandong 266011, P.R. China
}

Received July 31, 2018; Accepted January 28, 2019

DOI: $10.3892 / \mathrm{ol} .2019 .10073$

\begin{abstract}
The expression levels of thioredoxin (Trx), cytokeratin fragment 21-1 (CYFRA21-1) and serum squamous cell carcinoma antigen (SCCA) in patients with lung cancer and the diagnostic value of combined detection were investigated. Sixty-five patients with lung cancer in Weihai Municipal Hospital from January 2014 to June 2017 were retrospectively selected as the observation group, while 60 healthy subjects receiving physical examination were selected as the control group. The expression levels of serum Trx, CYFRA21-1 and SCCA were detected. The sensitivity and specificity of single detection and combined detection of these indexes were compared. Moreover, the diagnostic values of single detection and combined detection were analyzed using the receiver operating characteristic (ROC) curve. The levels of CYFRA21-1 and SCCA were the highest in squamous carcinoma $(\mathrm{P}<0.05)$. The level of Trx was the highest in small cell lung cancer compared with those in squamous carcinoma and adenocarcinoma $(\mathrm{P}<0.05)$. The levels of serum Trx, CYFRA21-1 and SCCA in lung cancer patients in clinical stage III-IV were obviously higher than those in patients in clinical stage I-II $(\mathrm{P}<0.001)$. The positive rate of Trx was the highest in small cell lung cancer, and the positive rates of CYFRA21-1 and SCCA were the highest in squamous carcinoma compared with other cancers $(\mathrm{P}<0.05)$. The area under the ROC curve of combined detection of the three indexes was the largest. The optimal cut-off value of combined detection of the three indexes in lung cancer was 9.952 with the sensitivity of $86.2 \%$ and specificity of $75.0 \%$. The detection of serum Trx, CYFRA21-1 and SCCA is of great significance in the diagnosis, progression and pathological type of lung cancer, and combined detection can
\end{abstract}

Correspondence to: Dr Huaping Tang, Department of Pneumology, Qingdao Municipal Hospital, 1 Jiaozhou Road, Qingdao, Shandong 266011, P.R. China

E-mail: tqj8r7@163.com

Key words: Trx, CYFRA21-1, SCCA, lung cancer, diagnostic value improve both specificity and sensitivity, which is more conducive to the positive rate of diagnosis of lung cancer.

\section{Introduction}

Lung cancer has top ranking in the malignant tumors in the world, and there are approximately 1,820,000 incidence cases and approximately 386,000 deaths (more male deaths than female deaths) every year (1). Lung cancer is mainly caused by the surrounding environment, which is related to air pollution, smoking, heredity, chronic lung diseases and occupational factors. In the early stage of lung cancer, there is no obvious specificity, but most patients with lung cancer, at diagnosis, have lost the optimal opportunity for treatment due to the rapid progression and high malignant degree of lung cancer (2). If changes in some tumor markers can be detected in the early stage of lung cancer, 'early detection and early treatment' can be realized, which is an important measure to reduce the mortality rate of lung cancer (3). Therefore, it is extremely important to search for lung cancer markers with high sensitivity and specificity.

Lung cancer markers mainly include hormones, enzymes, alpha-fetoprotein, cell surface membrane antigens and some cytokines (4). Thioredoxin (Trx) is a dimeric selenium-enzyme and a member of the pyridine nucleotide-disulfide oxidoreductase family, which is closely related to the condition of patients with lung cancer. Previous studies have demonstrated that Trx plays an important role in the development of cancer cells, and it is expected to be a new target for diagnosis, treatment and prognosis of tumor (5). Cytokeratin fragment 21-1 (CYFRA21-1) is a cytokeratin released into the blood in case of necrosis or lysis of tumor cells, its sensitivity and concentration increase with the progression of cancer cells. Therefore, CYFRA21-1 is considered as one of the best tumor markers for detecting lung cancer (6). Serum squamous cell carcinoma antigen (SCCA) is a tumor-associated antigen, and its levels in serum and specificity are higher in lung cancer patients (7). No lung cancer marker with good specificity and sensitivity has been found yet. Therefore, the combined detection of multiple tumor markers can complement one another and make up for deficiencies, thus providing an important auxiliary basis for the diagnosis and progression of lung cancer (8). In this study, the diagnostic value of combined detection of serum Trx, CYFRA21-1 and SCCA in lung cancer was investigated. 
Table I. Clinical data of patients [n (\%)].

\begin{tabular}{|c|c|c|c|c|}
\hline Groups & $\begin{array}{l}\text { Observation } \\
\text { group }(n=65)\end{array}$ & $\begin{array}{c}\text { Control } \\
\text { group }(n=60)\end{array}$ & $\chi^{2} / \mathrm{t}$ value & P-value \\
\hline Sex & & & 1.346 & 0.285 \\
\hline Male & $36(55.38)$ & $27(45.00)$ & & \\
\hline Female & $29(44.62)$ & $33(55.00)$ & & \\
\hline Age & & & 2.187 & 0.108 \\
\hline$\geq 55$ & $41(63.08)$ & $29(48.33)$ & & \\
\hline$<55$ & $24(36.92)$ & $31(51.67)$ & & \\
\hline Smoking & & & 0.157 & 0.593 \\
\hline Yes & $38(58.46)$ & $32(53.33)$ & & \\
\hline No & $27(41.54)$ & $28(46.67)$ & & \\
\hline Drinking & & & 0.936 & 0.285 \\
\hline Yes & $37(56.92)$ & $28(46.67)$ & & \\
\hline No & $28(43.08)$ & $32(53.33)$ & & \\
\hline Body mass index $\left(\mathrm{kg} / \mathrm{m}^{2}\right)$ & $21.3 \pm 3.9$ & $29.6 \pm 4.3$ & 1.433 & 0.154 \\
\hline
\end{tabular}

\section{Materials and methods}

Clinical data. A total of 65 patients with lung cancer in Weihai Municipal Hospital (Weihai, China) from January 2014 to June 2017 were selected as the observation group, including

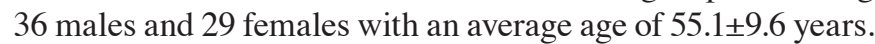
There were 30 cases of squamous carcinoma, 20 cases of adenocarcinoma and 15 cases of small cell lung cancer. Another 60 healthy subjects receiving physical examination were selected as the control group, including 36 males and 24 females with an average age of $52.1 \pm 7.2$ years.

Inclusion and exclusion criteria. Inclusion criteria: Patients clinically diagnosed with lung cancer, patients aged $\geq 18$ years, patients who had not undergone systematic treatment, and patients without other hereditary diseases. Exclusion criteria: Patients with diseases of the respiratory system, patients with cardiovascular or cerebrovascular diseases, handicapped patients, patients infected with the human immunodeficiency virus (HIV), or patients who had taken antibiotics in the prior three months. This study was approved by the Ethics Committee of Weihai Municipal Hospital. Subjects of the study were informed, agreed to participate in the clinical study and signed the informed consent.

Methods. After $5 \mathrm{ml}$ fasting venous blood was drawn from patients and healthy volunteers, it was centrifuged at 2,100 x g for $15 \mathrm{~min}$ at $4^{\circ} \mathrm{C}$ using a low-temperature high-speed centrifuge to collect the serum. The levels of serum CYFRA21-1 and SCCA were detected via chemiluminescence using the full-automatic chemiluminescence immune analyzer (MKY) and corresponding reagents. The level of Trx in cells was detected via enzyme-linked immunosorbent assay (ELISA) with human thioredoxin, Trx ELISA Kit (Cusabio, Wuhan, China) using a microplate reader (LB942; Berthold, Bad Wildbad, Germany) and corresponding reagents. The activity of Trx reductase was analyzed. Samples were collected and instruments were used strictly according to the manufacturer's instructions.

Evaluation criteria. The positive evaluation criteria for the three tumor markers are as follows: SCCA $>15 \mu \mathrm{g} / \mathrm{l}$, CYFRA21-1 $>50 \mu \mathrm{g} / 1$, and the optical density (OD) value of Trx samples $\geq$ cut-off value indicated positive.

Statistical analysis. Statistical Product and Service Solutions (SPSS) 17.0 (supported by Beijing Xinmei Jiahong Technology Co., Ltd., Beijing, China) was used for the analysis of all data in this experiment. Measurement data were expressed as mean \pm SD. $t$-test was used for the comparison between the two groups, and multi-factor analysis of variance was used for the comparison among the groups and the post hoc test was Dunnett's test. Enumeration data were expressed as rate (\%), and Chi-square test was adopted. The diagnostic values were analyzed using the ROC curve. $\mathrm{P}<0.05$ was considered to indicate a statistically significant difference.

\section{Results}

Analysis of clinical data of patients. Analysis of sex, age, smoking or not, drinking or not and body mass index in the observation and control groups revealed that there were no statistically significant differences in the groups $(\mathrm{P}>0.05)$ (Table I).

Determination of serum Trx, CYFRA21-1 and SCCA in the observation and control groups. The expression levels of serum Trx, CYFRA21-1 and SCCA in the observation group were, respectively, 47.6 $\pm 10.7,76.6 \pm 10.4$ and $45.3 \pm 6.9 \mu \mathrm{g} / 1$, which were higher than those in the control group (13.1 \pm 8.9 , $3.5 \pm 2.8$ and $7.8 \pm 3.2 \mu \mathrm{g} / \mathrm{l})(\mathrm{P}<0.01)$ (Fig. 1).

Diagnostic value analysis of single and combined detection of Trx, CYFRA21-1 and SCCA. The area under the receiver 
A

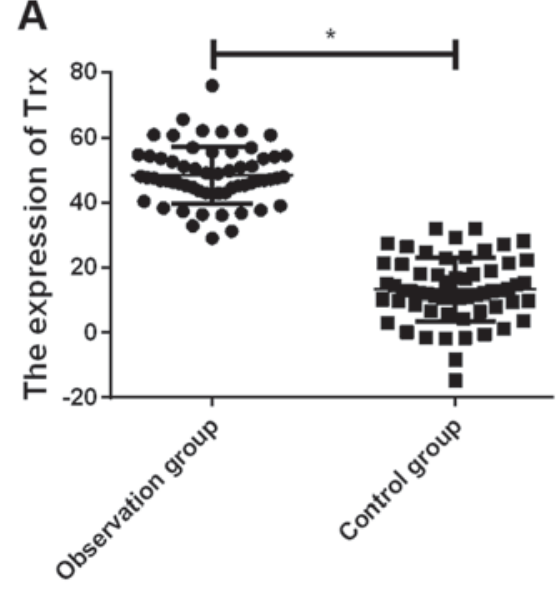

B

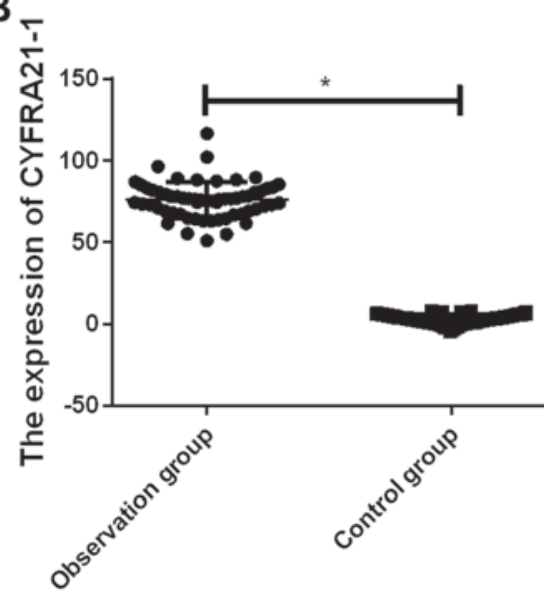

C

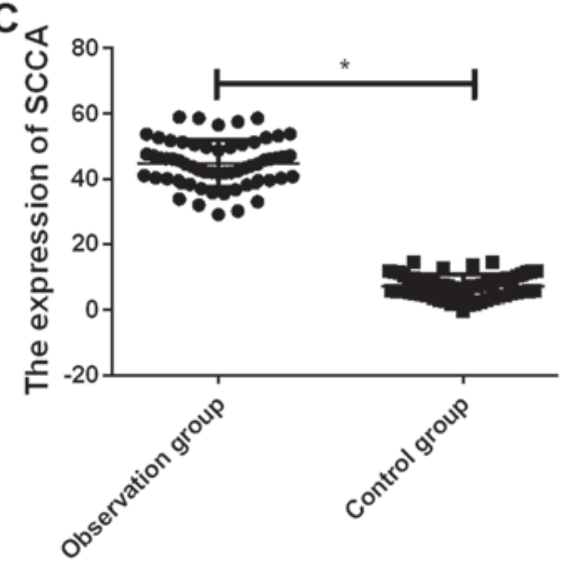

Figure 1. Trx, CYFRA21-1 and SCCA expression levels. (A) The expression level of Trx in the observation group is significantly higher than that in the control group, and there is a statistically significant difference ( $\left.{ }^{*} \mathrm{P}<0.05\right)$. (B) The expression level of CYFRA21-1 in the observation group is significantly higher than that in the control group, and there is a statistically significant difference $\left({ }^{*} \mathrm{P}<0.05\right)$. (C) The expression level of SCCA in the observation group is obviously higher than that in the control group, displaying a statistically significant difference $\left({ }^{*} \mathrm{P}<0.05\right)$.

Table II. ROC curve analysis of single and combined detection of Trx, CYFRA21-1 and SCCA.

\begin{tabular}{lcccccc}
\hline Index & Area under curve & OR & 95\%CI & P-value & Sensitivity $(\%)$ & Specificity $(\%)$ \\
\hline Trx & 0.674 & 0.048 & $0.580-0.768$ & 0.001 & 58.5 & 73.3 \\
CYFRA21-1 & 0.829 & 0.037 & $0.757-0.901$ & 0.001 & 70.8 & 85.0 \\
SCCA & 0.684 & 0.048 & $0.590-0.779$ & 0.001 & 64.6 & 76.0 \\
Combined detection & 0.851 & 0.035 & $0.782-0.919$ & 0.001 & 86.2 & 75.0 \\
\hline
\end{tabular}

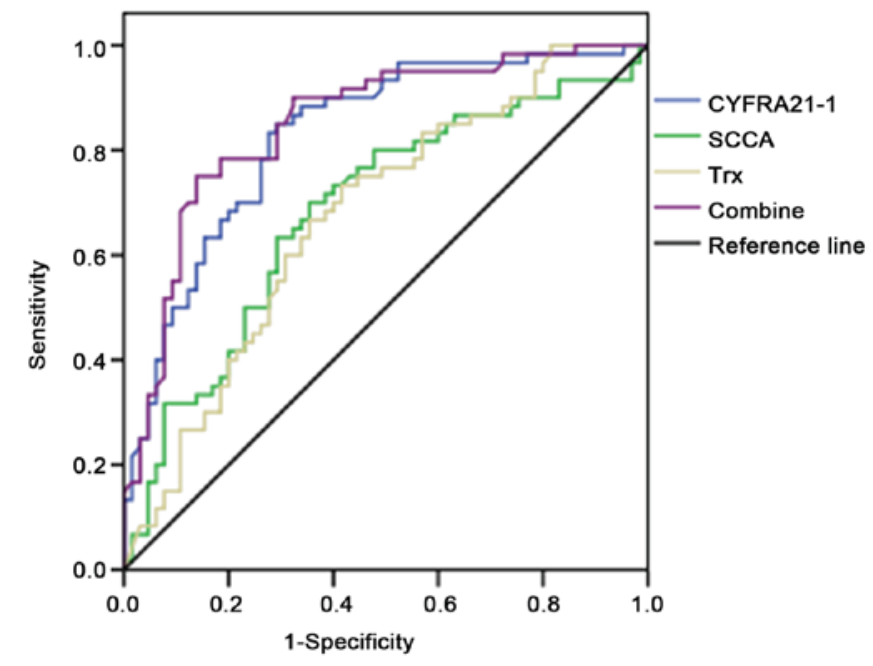

Figure 2. ROC curve analysis. The area under the curve of combined detection of the three indexes is the largest, followed by CYFRA21-1, SCCA and Trx, showing statistically significant differences $(\mathrm{P}<0.05)$.

operating characteristic (ROC) curve of combined detection of the three indexes was the largest, followed by CYFRA21-1, SCCA and Trx. The value of combined detection of the three indexes was higher than that of single detection, and the sensitivity of combined detection of the three indexes was obviously higher than that of single detection, displaying statistically significant differences $(\mathrm{P}<0.05)$. The combined detection of the three indexes in lung cancer was the best with the sensitivity of $86.2 \%$ and specificity of $75.0 \%$ (Table II and Fig. 2).

Comparison of serum Trx, CYFRA21-1 and SCCA levels in different pathological types of lung cancer. The levels of CYFRA21-1 and SCCA were the highest in squamous carcinoma, and there were statistically significant differences compared with those in adenocarcinoma and small cell lung cancer $(\mathrm{P}<0.05)$. The level of Trx was the highest in small cell lung cancer, and it had statistically significant differences compared with those in squamous carcinoma and adenocarcinoma $(\mathrm{P}<0.05)$ (Table III).

Comparison of serum Trx, CYFRA21-1 and SCCA levels in different clinical stages of lung cancer. The levels of serum Trx, CYFRA21-1 and SCCA in lung cancer patients in clinical stage III-IV were obviously higher than those in patients in clinical stage I-II, displaying statistically significant differences $(\mathrm{P}<0.001)$. There were no statistically significant differences in levels of Trx, CYFRA21-1 and SCCA in patients with lymph node metastasis and distant metastasis $(\mathrm{P}>0.05)$ (Table IV).

Comparison of positive rates of Trx, CYFRA21-1 and SCCA in different pathological types of lung cancer. The positive rate of Trx was the highest in small cell lung cancer, and the positive rates of CYFRA21-1 and SCCA were the highest in 
Table III. Comparison of serum Trx, CYFRA21-1 and SCCA levels in different pathological types of lung cancer.

\begin{tabular}{lcccc}
\hline Pathological types & $\mathrm{n}$ & $\operatorname{Trx}(\mu \mathrm{g} / \mathrm{l})$ & CYFRA21-1 $(\mu \mathrm{g} / \mathrm{l})$ & SCCA $(\mu \mathrm{g} / \mathrm{l})$ \\
\hline Squamous carcinoma & 30 & $22.6 \pm 21.1$ & $95.9 \pm 23.9^{\mathrm{b}}$ & $101.8 \pm 29.8^{\mathrm{b}}$ \\
Adenocarcinoma & 20 & $18.6 \pm 10.8$ & $39.9 \pm 10.6$ & $17.6 \pm 10.2$ \\
Small cell lung cancer & 15 & $56.9 \pm 8.7^{\mathrm{a}}$ & $30.5 \pm 10.6$ & $17.5 \pm 8.9$ \\
F value & & 12.77 & 10.32 & 14.64 \\
P-value & 0.001 & 0.001 & 0.001 \\
\hline
\end{tabular}

${ }^{\text {a }} \mathrm{P}<0.01$ vs. squamous carcinoma and adenocarcinoma, and ${ }^{\mathrm{b}} \mathrm{P}<0.01$ vs. adenocarcinoma and small cell lung cancer.

Table IV. Comparison of serum Trx, CYFRA21-1 and SCCA levels in different clinical stages of lung cancer.

\begin{tabular}{lcccc}
\hline Groups & $\mathrm{n}$ & Trx & CYFRA21-1 & SCCA \\
\hline Stage I-II & 20 & $17.9 \pm 5.7$ & $16.5 \pm 4.9$ & $14.3 \pm 5.6$ \\
Stage III-IV & 45 & $30.9 \pm 6.1$ & $28.6 \pm 8.4$ & $25.5 \pm 6.3$ \\
t value & & 8.086 & 5.989 & 6.835 \\
P-value & & 0.001 & 0.001 & 0.001 \\
Lymph node metastasis & 28 & $26.3 \pm 5.3$ & $21.6 \pm 2.5$ & $24.8 \pm 4.3$ \\
Distant metastasis & 25 & $24.5 \pm 4.2$ & $20.4 \pm 5.6$ & $25.6 \pm 5.9$ \\
t value & & 1.359 & 1.026 & 0.568 \\
P-value & & 0.180 & 0.310 & 0.572 \\
\hline
\end{tabular}

Table V. Comparison of positive rates of Trx, CYFRA21-1 and SCCA in different pathological types of lung cancer [n (\%)].

\begin{tabular}{lcccc}
\hline Pathological types & $\mathrm{n}$ & Trx & CYFRA21-1 & SCCA \\
\hline Squamous carcinoma & 30 & $16(53.33)$ & $24(80.00)^{\mathrm{b}}$ & $26(86.67)^{\mathrm{c}}$ \\
Adenocarcinoma & 25 & $9(52.00)$ & $9(36.00)$ & $10(40.00)$ \\
Small cell lung cancer & 10 & 4.851 & $4(40.00)$ & $6(60.00)$ \\
$\chi^{2}$ value & & 0.050 & 12.147 & 13.099 \\
P-value & & 0.002 & 0.001
\end{tabular}

${ }^{\mathrm{a}} \mathrm{P}<0.05$ vs. squamous carcinoma and adenocarcinoma, ${ }^{\mathrm{b}} \mathrm{P}<0.05$ vs. adenocarcinoma and small cell lung cancer, and ${ }^{\mathrm{c}} \mathrm{P}<0.05$ vs. small cell lung cancer and adenocarcinoma.

squamous carcinoma, showing statistically significant differences compared with other cancers $(\mathrm{P}<0.05)$ (Table V).

\section{Discussion}

The incidence and mortality rates of lung cancer have shown increasing trends in recent years, seriously threatening people's health. However, it is noteworthy that there are generally no obvious symptoms in the early stage of lung cancer, and it is hard for patients to notice. At the same time, rich blood supply in lung tissues provides favourable conditions for the metastasis of cancer cells, so that patients are often in the late stage once there are obvious clinical symptoms, leading to difficult treatment, which is also an important cause of the high mortality rate of lung cancer (9). Therefore, the early diagnosis and treatment of lung cancer are of great importance. In the diagnosis of lung cancer, tissue biopsy serves as a golden standard in clinic, but it is only applied when symptoms develop and patients need to be diagnosed, and cannot be used in the early screening of lung cancer due to trauma (10). Therefore, the value of tumor markers in the diagnosis of early lung cancer has gained extensive attention.

The oxidative active amino acid composition of $\operatorname{Tr} x$ is Cys-Gly-Pys-Cys, and Trx can catalyze the redox reaction and also maintain intracellular homeostasis, regulate apoptosis and resist oxidative stress response under biological functions (11). A number of studies have demonstrated that the expression of Trx is increased in a variety of malignant tumors, such as gastric, breast, liver, lung, rectal and cervical cancers $(7,12)$, indicating that $\operatorname{Trx}$ is closely related to malignant tumors. It was found in related studies that Trx can inhibit apoptosis of tumor cells, promote cell proliferation and disturb normal 
cell cycle (13). CYFRA21-1 is a cytokeratin existing in cells of epithelium-derived malignant tumors. A large amount of soluble CYFRA21-1 enters the blood circulation in case of lysis or death of tumor cells (14). Moreover, studies have revealed that the expression level of serum CYFRA21-1 in patients with lung cancer is remarkably higher than that in healthy individuals, so it can be used in the identification of malignant lesions in the lung (15). SCCA is a tissue antigen, which is mainly used in the diagnosis of squamous carcinoma, including squamous carcinoma in the head and neck, lung and trachea, esophagus, cervix, anal canal, genitourinary tract and brain, monitoring of condition and prognosis and therapeutic evaluation (16).

In this study, it was found that the expression levels of serum Trx, CYFRA21-1 and SCCA in the observation group were higher than those in the control group $(\mathrm{P}<0.01)$, and there were significant differences between the two groups, indicating that the tumor markers (Trx, CYFRA21-1 and SCCA) have important value in the clinical diagnosis of lung cancer. The above results are basically consistent with those in related results, and the more severe the disease is, the higher the levels of tumor markers are (17), suggesting that the levels of serum Trx, CYFRA21-1 and SCCA can provide a certain reference basis for the diagnosis of lung cancer. In this study, the levels of CYFRA21-1 and SCCA were the highest in squamous carcinoma, and there were statistically significant differences compared with those in adenocarcinoma and small cell lung cancer $(\mathrm{P}<0.05)$. The level of Trx was the highest in small cell lung cancer, and it had statistically significant differences compared with those in squamous carcinoma and adenocarcinoma $(\mathrm{P}<0.05)$, suggesting that CYFRA21-1 and SCCA are superior in detecting squamous carcinoma to adenocarcinoma and small cell lung cancer, and Trx is more valuable in detecting small cell lung cancer. SCCA is a good marker for the diagnosis of squamous carcinoma, which can monitor the disease development and evaluate the prognosis. Trx, CYFRA21-1 and SCCA are of important significance in guiding the diagnosis and treatment of lung squamous carcinoma. The three indexes can improve the diagnostic accuracy rate of lung cancer and help provide diagnostic information, so as to perform the targeted treatment, improve the therapeutic efficiency and prolong the life of patients, which are basically consistent with results in related studies (18). In this study, the levels of serum Trx, CYFRA21-1 and SCCA in lung cancer patients in clinical stage III-IV were obviously higher than those in patients in clinical stage I-II, indicating that Trx, CYFRA21-1 and SCCA are related to the progression of lung cancer, and they can be used to monitor metastasis and recurrence of malignant tumor in clinic. The concentration of serum tumor markers is positively correlated with the tumor size, which is also consistent with related literature reports (19). Results of this study manifested that despite high specificity of single detection of Trx, CYFRA21-1 and SCCA, the sensitivity is unsatisfactory, failing to meet the clinical requirements. Therefore, lung cancer should not be diagnosed using only one single index at present, but multiple indexes should be combined for joint diagnosis, thus improving the accuracy. The sensitivity of combined detection of the three indexes is improved, and the sensitivity, specificity and accuracy are $86.8,80.4$ and $89.6 \%$, respectively. It can be seen that both sensitivity and specificity are significantly improved compared with single detection, thus benefiting the early screening of lung cancer patients, performing the treatment as early as possible improves the prognosis, which is consistent with the report of Jiang et al (20). Combined detection plays an important role in the diagnosis of lung cancer, which can be used in the early screening of patients with lung cancer. The ROC curve can reflect the specificity, sensitivity and accuracy of detection indexes objectively and accurately. The closer the area under the curve is to 0.5 , the lower the diagnostic accuracy will be, and the closer it is to 1.0, the higher the diagnostic accuracy will be. In this study, the area under the ROC curve of combined detection of the three indexes was the largest, followed by CYFRA21-1, SCCA and Trx. The area under the curve was $<0.9$ in single detection of the three indexes and 0.906 in combined detection, and the combined detection of the three indexes in lung cancer was optimal with the sensitivity of $86.2 \%$ and specificity of $75.0 \%$, proving that both accuracy and efficiency of combined detection are superior to those of single detection of any index, which are consistent with results of Zhang et al (21). The above results suggest that the combined detection is able to improve the diagnostic rate.

The combined detection of the three indexes can improve the accuracy and can also be used in the early screening and early treatment of lung cancer and the improvement of prognosis. However, there are still some false-negative and false-positive cases in the combined detection of the three indexes in the diagnosis of lung cancer, indicating that lung cancer still cannot be diagnosed only using one means, but imaging and pathological methods should be combined for comprehensive evaluation, thereby avoiding misdiagnosis and improving diagnostic accuracy. The sample size in this study was small, and there was a certain selection bias, so further study is still necessary for verification.

In conclusion, the detection of serum Trx, CYFRA21-1 and SCCA is of great significance in the diagnosis, progression and pathological type of lung cancer, and combined detection can improve both specificity and sensitivity, which is more conducive to the positive rate of diagnosis of lung cancer.

\section{Acknowledgements}

Not applicable.

\section{Funding}

No funding was received.

\section{Availability of data and materials}

The datasets used and/or analyzed during the present study are available from the corresponding author on reasonable request.

\section{Authors' contributions}

TQ, JZ, NX and BL collected and analyzed the general data of patients. ML and ALiu performed ELISA. ALi and HT evaluated the tumor markers. All the authors read and approved the final manuscript. 


\section{Ethics approval and consent to participate}

The study was approved by the Ethics Committee of Weihai Municipal Hospital (Weihai, China). Signed informed consents were obtained from the patients and/or guardians.

\section{Patient consent for publication}

Not applicable.

\section{Competing interests}

The authors declare that they have no competing interests.

\section{References}

1. Ferlay J, Soerjomataram I, Dikshit R, Eser S, Mathers C, Rebelo M, Parkin DM, Forman D and Bray F: Cancer incidence and mortality worldwide: Sources, methods and major patterns in GLOBOCAN 2012. Int J Cancer 136: E359-E386, 2015.

2. D'Addario G, Früh M, Reck M, Baumann P, Klepetko W and Felip E; ESMO Guidelines Working Group: Metastatic non-small-cell lung cancer: ESMO Clinical Practice Guidelines for diagnosis, treatment and follow-up. Ann Oncol 21 (Suppl 5): v116-v119, 2010.

3. Vansteenkiste J, Crinò L, Dooms C, Douillard JY, Faivre-Finn C, Lim E, Rocco G, Senan S, Van Schil P, Veronesi G, et al; Panel Members: 2nd ESMO Consensus Conference on Lung Cancer: Early-stage non-small-cell lung cancer consensus on diagnosis, treatment and follow-up. Ann Oncol 25: 1462-1474, 2014.

4. Boonstra MC, de Geus SW, Prevoo HA, Hawinkels LJ, van de Velde CJ, Kuppen PJ, Vahrmeijer AL and Sier CF: Selecting targets for tumor imaging: An overview of cancerassociated membrane proteins. Biomark Cancer 8: 119-133, 2016.

5. Liang B, Shao W, Zhu C, Wen G, Yue X, Wang R, Quan J, Du J and Bu X: Mitochondria-targeted approach: remarkably enhanced cellular bioactivities of TPP2a as selective inhibitor and probe toward TrxR. ACS Chem Biol 11: 425-434, 2016.

6. Negrin LL, Halat G, Kettner S, Gregori M, Ristl R, Hajdu S and Heinz T: Club cell protein 16 and cytokeratin fragment 21-1 as early predictors of pulmonary complications in polytraumatized patients with severe chest trauma. PLoS One 12: e0175303, 2017.

7. Holdenrieder S, Molina R, Qiu L, Zhi X, Rutz S, Engel C, Kasper-Sauer P, Dayyani F and Korse CM: Technical and clinical performance of a new assay to detect squamous cell carcinoma antigen levels for the differential diagnosis of cervical, lung, and head and neck cancer. Tumour Biol 40: 1010428318772202, 2018.

8. Nakamura $\mathrm{H}$ and Nishimura T: History, molecular features, and clinical importance of conventional serum biomarkers in lung cancer. Surg Today 47: 1037-1059, 2017.

9. Shen YW, Zhang XM, Li ST, Lv M, Yang J, Wang F, Chen ZL, Wang BY, Li P, Chen L, et al: Efficacy and safety of icotinib as first-line therapy in patients with advanced non-small-cell lung cancer. OncoTargets Ther 9: 929-935, 2016.
10. He WJ, Li WH, Jiang B, Wang YF, Xia YX and Wang L: MicroRNAs level as an initial screening method for early-stage lung cancer: A bivariate diagnostic random-effects meta-analysis. Int J Clin Exp Med 8: 12317-12326, 2015.

11. Arjune S, Schwarz G and Belaidi AA: Involvement of the Cys-Tyr cofactor on iron binding in the active site of human cysteine dioxygenase. Amino Acids 47: 55-63, 2015.

12. Zheng X, Xu W, Sun R, Yin H, Dong C and Zeng H: Synergism between thioredoxin reductase inhibitor ethaselen and sodium selenite in inhibiting proliferation and inducing death of human non-small cell lung cancer cells. Chem Biol Interact 275: 74-85, 2017.

13. You BR, Kim SH and Park WH: Reactive oxygen species, glutathione, and thioredoxin influence suberoyl bishydroxamic acid-induced apoptosis in A549 lung cancer cells. Tumour Biol 36: 3429-3439, 2015.

14. Dohmoto K, Hojo S, Fujita J, Yang Y, Ueda Y, Bandoh S, Yamaji Y, Ohtsuki Y, Dobashi N, Ishida T, et al: The role of caspase 3 in producing cytokeratin 19 fragment (CYFRA21-1) in human lung cancer cell lines. Int J Cancer 91: 468-473, 2001.

15. Liu L, Teng J, Zhang L, Cong P, Yao Y, Sun G, Liu Z, Yu T and Liu M: The combination of the tumor markers suggests the histological diagnosis of lung cancer. BioMed Res Int 2017: 2013989, 2017.

16. Zhao W, Yu H, Han Z, Gao N, Xue J and Wang Y: Clinical significance of joint detection of serum CEA, SCCA, and bFGF in the diagnosis of lung cancer. Int J Clin Exp Pathol 8: 9506-9511, 2015

17. Chen F, Wang XY, Han XH, Wang H and Qi J: Diagnostic value of Cyfra21-1, SCC and CEA for differentiation of early-stage NSCLC from benign lung disease. Int $\mathrm{J}$ Clin Exp Med 8: 11295-11300, 2015.

18. Chen MS, Xu Y, Ma J, Wu CG, Hao XK, Lu BB and Liu T: Relation between the level of TPS, NSE, CEA and beta2-mG in the serum and the biological behavior of small cell lung cancer. Xi Bao Yu Fen Zi Mian Yi Xue Za Zhi 23: 751-753, 2007 (In Chinese).

19. Schmidt B, Beyer J, Dietrich D, Bork I, Liebenberg V and Fleischhacker M: Quantification of cell-free mSHOX2 Plasma DNA for therapy monitoring in advanced stage non-small cell (NSCLC) and small-cell lung cancer (SCLC) patients. PLoS One 10: e0118195, 2015

20. Jiang ZF, Wang $M$ and Xu JL: Thymidine kinase 1 combined with CEA, CYFRA21-1 and NSE improved its diagnostic value for lung cancer. Life Sci 194: 1-6, 2018.

21. Zhang L, Liu D, Li L, Pu D, Zhou P, Jing Y, Yu H, Wang Y, Zhu Y, He Y, et al: The important role of circulating CYFRA21-1 in metastasis diagnosis and prognostic value compared with carcinoembryonic antigen and neuron-specific enolase in lung cancer patients. BMC Cancer 17: 96, 2017.

This work is licensed under a Creative Commons Attribution-NonCommercial-NoDerivatives 4.0 International (CC BY-NC-ND 4.0) License. 